\title{
Biotinidase deficiency: a survey of 10 cases
}

\author{
H J WASTELL, ${ }^{*} \mathrm{~K}$ BARTLETT, $\dagger$ G DALE, ${ }^{*}$ AND A SHEIN \\ *Department of Clinical Biochemistry, Newcastle General Hospital, and tDepartments of Child Health \\ and Clinical Biochemistry, Newcastle University Medical School, Newcastle upon Tyne
}

SUMmARY Ten patients with biotinidase deficiency were studied. Clinical findings at presentation varied with dermatological signs (dermatitis and alopecia), neurological abnormalities (fits, hypotonia, and ataxia), and recurrent infections being the most common features, although none of these occurred in every case. Biochemically the disease is characterised by metabolic acidosis and organic aciduria. Treatment with biotin results in pronounced, rapid, clinical and biochemical improvement, but some patients have residual neurological damage comprising neurosensory hearing loss, visual pathway defects, ataxia, and mental retardation. The cause of this permanent damage remains obscure and it is not clear if the early introduction of treatment will prevent it.

Biotin is a cofactor required by acetyl CoA carboxylase (ACC) [EC 6.4.1.2], pyruvate carboxylase (PC) [EC 6.4.1.1], propionyl CoA carboxylase (PCC) [EC 6.4.1.3] and 3 methylcrotonyl $\mathrm{CoA}$ carboxylase (MCC) [EC 6.4.1.4.]. ${ }^{1}$ It is covalently attached to the apocarboxylases by the epsilon amino group of a lysine residue, where it functions at the active site as a carbon dioxide carrier in the carboxylation reactions. Biotinylation of the apocarboxylases is catalysed by holocarboxylase synthetase in an adenosine triphosphate dependent reaction with the intermediate formation of biotinyl adenosine monophosphate. ${ }^{2}$ The turnover of carboxylases yields biotinyllysine (biocytin) from which biotin is regenerated by the action of a specific amidolyase, biotinidase [EC 3.5.1.12]. This enzyme is also required for the release of dietary protein bound biotin.

Individual inherited disorders of all four biotin dependent carboxylases have been reported. ${ }^{3-9}$ In addition, patients with simultaneous defects of all four enzymes (combined carboxylase deficiency) who showed remarkable clinical and biochemical responses to pharmacological doses of biotin have been described. In some patients (usually neonates) this biotin responsiveness is also expressed in cultured cells whereas in others (who usually present later) no defect can be found. In the majority of patients with late onset combined carboxylase deficiency the underlying defect is biotinidase deficiency. ${ }^{10}$ The early onset form, on the other hand, is due to holocarboxylase synthetase deficiency. ${ }^{11}$ Thus combined carboxylase deficiency can be caused by either a functional biotin deficiency (biotinidase deficiency) caused by failure to recycle endogenous biotin and to liberate dietary biotin, or by defective biotinylation of apocarboxylase because of a mutant holocarboxylase synthetase that has an increased $\mathrm{K}_{\mathrm{m}}$ with respect to biotin. ${ }^{12}$ In both these inborn errors, treatment with pharmacological doses of biotin may correct the disorder.

Holocarboxylase deficiency usually presents acutely in the neonatal period but occasionally becomes apparent later. It is characterised by severe metabolic acidosis, organic aciduria, hyperammonaemia, lethargy, hypotonia, and vomiting. Biotinidase deficiency shows a less consistent clinical picture and Wolf $e t$ al $^{13}$ documented the phenotypic variations though some of the cases were misassigned. ${ }^{14}$ The occurrence of persistent auditory and visual defects is now well recognised. ${ }^{13} 1516$

We present our findings in 10 cases of definite biotinidase deficiency and suggest that although there is complete resolution of the biochemical abnormalities, there may be residual defects in some patients.

\section{Case reports}

Symptoms at presentation and long term complications are summarised in the table.

CASE 1

This patient was first described by Bartlett $e t$ al (case 3). ${ }^{17}$ She was born in 1974 weighing $3350 \mathrm{~g}$, the first child of related Irish parents; she has a younger 
Table Clinical presentation and outcome of 10 cases of biotinidase deficiency

\begin{tabular}{|c|c|c|c|c|c|c|c|c|c|c|}
\hline & \multicolumn{10}{|c|}{ Case No } \\
\hline & 1 & 2 & 3 & 4 & 5 & 6 & 7 & $8^{*}$ & 9 & 10 \\
\hline Age at onset (months) & 5 & 10 & 3 & $2 \cdot 5$ & 7 & 1.5 & 8 & * & 18 & 18 \\
\hline Age treatment started (months) & 10 & 15 & 22 & 8 & $7 \cdot 5$ & 17 & $9 \cdot 5$ & $2 \cdot 5$ & 24 & 24 \\
\hline \multicolumn{11}{|l|}{ Symptoms: } \\
\hline Ataxia & No & Yes & Yes & No & No & Yes & Yes & * & Yes & Yes \\
\hline Hypotonia & Yes & Yes & Yes & No & No & Yes & Yes & $*$ & Yes & Yes \\
\hline Fits & No & No & Yes & Yes & Yes & No & Yes & * & No & No \\
\hline Developmental delay & No & No & No & Yes & No & Yes & No & * & No & No \\
\hline Alopecia & Yes & Yes & Yes & Yes & No & Yes & Yes $\dagger$ & $*$ & No & Yes \\
\hline Dermatitis & No & Yes & No & Yes & No & Yes & No & $*$ & No & Yes \\
\hline Respiratory infections & Yes & Yes & Yes & Yes & No & Yes & Yes & * & Yes & No \\
\hline \multicolumn{11}{|l|}{ Long term complications: } \\
\hline Auditory & Yes $\dagger$ & Yes $\dagger$ & Yes $\dagger$ & No & No & No & Yes $\dagger$ & No & Yes $\dagger$ & No \\
\hline Visual & No & Yes $\dagger$ & Yes & No & No & No & No & No & No & Yes \\
\hline Intellectual & No & No & Yes & No & No & Yes & No & No & No & No \\
\hline Ataxia & No & No & No & No & No & No & Yes & Yes $\dagger$ & Yes & No \\
\hline
\end{tabular}

${ }^{*}$ Receiving presymptomatic treatment: †symptoms diagnosed or presenting during treatment.

sister who is well. She developed normally until she was 5 months old when, after an upper respiratory tract infection, she was noted to be hyperventilating. This persisted and at the age of 8 months she was admitted to hospital for investigation. During the next two months she became so hypotonic that virtually all spontaneous movement ceased. She had a persistent compensated metabolic acidosis, and urinary organic acid analysis at the age of 10 months showed large amounts of 3-hydroxyisovaleric acid and 3-methylcrotonylglycine. Based on these findings a diagnosis of MCC deficiency was made and treatment with biotin $(5 \mathrm{mg}$ twice a day, later reduced to $5 \mathrm{mg}$ daily) was started. Within 24 hours the hyperventilation stopped, and by the end of a week there had been considerable improvement in the hypotonia. When reviewed at the age of $11 \frac{1}{2}$ months she was alert, could sit unsupported, reach out, and play.

At the age of 20 months she was thought to be normal, but at the age of $2 \frac{1}{2}$ years her speech was delayed and she had a profound neurosensory hearing loss. Apart from juvenile glaucoma diagnosed at the age of 6 , development has otherwise been normal. Studies on cultured skin fibroblasts brought the diagnosis of MCC deficiency into doubt as the activities of two of the carboxylases were normal ( $R$ Chalmers, personal communication). Biotinidase activity was later undetectable.

\section{CASE 2}

This patient has already been described by Taitz et $a l$, Charles et al, and Taitz et al (case 1 in each report). ${ }^{1618}{ }^{19}$ He was born in 1977 weighing $2900 \mathrm{~g}$, the second child of unrelated English parents; his older sister is normal. Although he suffered from recurrent respiratory tract and eye infections he progressed normally until the age of 10 months when he developed a rash and alopecia, and became unsteady with truncal ataxia. Acid base balance at that time was normal.

By the age of 15 months he had regressed to a developmental age of 1 month and was profoundly hypotonic. At this time a metabolic acidosis was noted; plasma lactate was raised $(8.9 \mathrm{mmol} / \mathrm{l}$, normal range $0.56-2.2 \mathrm{mmol} / \mathrm{l})$ and urinary organic acid analysis showed excessive amounts of 3-hydroxyisovalerate, 3-methylcrotonylglycine, methylcitrate, and 3-hydroxypropionate.

$\mathrm{He}$ was given biotin $10 \mathrm{mg}$ a day with remarkable effect. His neurological state returned to normal within three days, the dermatosis, alopecia, and recurrent eye infections resolved, and the respiratory infections improved. He now has a neurosensory hearing loss as well as a conductive hearing loss secondary to glue ear, and profound myopia with evidence of acquired retinal dysplasia. These defects were not detected before the biotin was started and they now seem to be stable as he has had no further deterioration in his visual or hearing acuity for four years. Development otherwise has been normal and at the age of 9 years he was making good progress intellectually, was in the upper stream in his class, and was thought to be well above average in his academic ability. Biotinidase was measured later; no activity was detected.

CASE 3

This patient was first described by Taitz et al (case 3). ${ }^{16} \mathrm{He}$ was born in 1979 weighing $2020 \mathrm{~g}$, the 
second child of unrelated Indian parents. Apart from being 'chesty' he was well until the age of 3 months when he developed convulsions that were not controlled by phenobarbitone. Acidosis was not noted. Development was normal during the first year but thereafter he began to regress. At 16 months he became unwell with a pyrexial illness, diarrhoea, vomiting, and a chest infection; he also developed alopecia. There was no note of acidosis.

At the age of 22 months he was admitted to hospital for investigation where he was found to be hypotonic and grossly ataxic, and had regressed to a developmental age of 11-12 months; he was bald and tachypnoeic with a chronic chest infection. Fundoscopy showed no abnormality, but visual evoked responses suggested the possibility of some loss of function of the visual pathways or cortex. Urinary organic acid analysis showed a large excess of 3-hydroxybutyrate and 3-hydroxyisovalerate. Leucocyte carboxylase activities were reduced (PCC $3.0 \%$, MCC $0.8 \%$, PC $0.8 \%$, and ACC $2 \cdot 3 \%$, expressed as percentages of the means of the normal ranges). Biotin $10 \mathrm{mg}$ a day was started and resulted in neurological improvement within days, his hair regrew, and the chest infections occurred less often. Leucocyte carboxylase activities returned to normal. His speech, however, did not progress and he was found to have a severe neurosensory deafness. His development is also generally mildly delayed. Biotinidase activity was subsequently undetectable.

\section{CASE 4}

This boy was born in 1979 weighing $1480 \mathrm{~g}$, the second child of related parents of Scottish tinker stock. An older sister is well and another child was stillborn at 34 weeks' gestation. He is related to two other children with biotinidase deficiency (case 6 in the present report and case 2 reported by Taitz et $\left.a l^{16}\right)$. He was delivered at 37 weeks' gestation by caesarean section for fetal distress preceded by intrauterine growth retardation. At 10 weeks he had a generalised convulsion that was treated with phenobarbitone. At the age of 3 months he had three more fits and the dose of phenobarbitone was increased. He was admitted to hospital at the age 5 months for repair of a strangulated hernia at which time it was noted that he was failing to thrive, and it was found that he had a history of recurrent upper respiratory tract infections, blepharitis, and staphlococcal skin infections. Alopecia, developmental delay, and regression were also noted and an intermittent compensated metabolic acidosis was found. At the age of 6 months the fits recurred and despite treatment with anticonvulsant drugs progressed to status epilepticus. The mild metabolic acidosis persisted with a raised plasma lactate concentration $(4.8 \mathrm{mmol} / \mathrm{l}$, normal range $0 \cdot 67-1 \cdot 7$ $\mathrm{mmol} / \mathrm{l})$. Urinary organic acid concentrations were normal. By the age of 7 months there was a constant profound metabolic acidosis and analysis of urinary organic acids revealed excessive excretion of 3hydroxyisovalerate and 3-methylcrotonylglycine. Leucocyte carboxylase activities were reduced (PCC, PC, and MCC all less than $1 \%$ of normal). Plasma biotin concentration was $0.3 \mathrm{mmol} / \mathrm{l}$ (normal 0.29-1.9) measured by an avidin binding assay that does not distinguish between biotin and biocytin. ${ }^{17}$

At the age of 8 months biotin $10 \mathrm{mg}$ a day was started and within four days it was possible to stop all anticonvulsant treatment. Leucocyte carboxylase activities returned to normal (PCC 63\%, MCC $113 \%$, and PCC $61 \%$, all activities expressed as percentage of the means of normal ranges) and the plasma biotin concentration was $113 \mathrm{mmol} / \mathrm{l}$.

At the age of $5 \frac{1}{2}$ years he appeared to be developing normally within his cultural and social background. Hearing and vision seemed normal, although formal testing was not done.

CASE 5

This girl was born in 1982 weighing $2730 \mathrm{~g}$, the third child of related Pakistani parents. One sibling died at the age of $4 \frac{1}{2} 2$ months of 'twitching', the other, 4 years older than the patient, was well at the time of writing.

She was admitted to hospital at the age of 7 months with tachypnoea of sudden onset and twitching of the face and limbs. She was found to be developmentally normal although unable to sit unsupported; tone and reflexes were normal. She had a persistent compensated metabolic acidosis (hydrogen ion concentration $39 \mathrm{nmol} / 1$, base deficit $-10 \mathrm{mmol} / \mathrm{l})$ and plasma concentrations of pyruvate and lactate were raised $(0.12 \mathrm{mmol} / \mathrm{l}$, normal range $0.041-0.067$, and $2.98 \mathrm{mmol} / \mathrm{l}$, normal range $1-1 \cdot 8$, respectively). The twitching ceased when the acidosis was corrected with bicarbonate. Five days later the twitching recurred and she became hypotonic with poor head control. A metabolic acidosis was noted (hydrogen ion concentration $40 \mathrm{nmol} / \mathrm{l}$, base deficit $-7.3 \mathrm{mmol} / \mathrm{l})$. Urinary organic acids were abnormal with a large excess of 3-hydroxyisovalerate, and concentrations of 3-methylcrotonylglycine, methylcitrate, and 3-hydroxypropionate. A provisional diagnosis of 3-methylcrotonylglycinuria was made and she was given biotin $5 \mathrm{mg}$ twice a day. She improved within four days, and three weeks later was able to sit unsupported. Since that time she has remained well with no evidence of developmental delay, or visual or auditory problems. Biotinidase deficiency was later proved. 
CASE 6

This girl was born in 1981 weighing $2810 \mathrm{~g}$, the first child of related Scottish tinkers and is related to case 4 in the present study and to another child with biotinidase deficiency (case 2 reported by Taitz et $\mathrm{al}^{16}$ ). She had recurrent stridor from the age of 6 weeks resulting in several admissions to hospital and at 16 months she was admitted acutely ill with a 24 hour history of fever, vomiting, stridor, and drowsiness; she was hypotonic and comatose with truncal ataxia, stridor, alopecia, and a rash. Her condition improved after intravenous fluid replacement but she had gross developmental delay (a developmental age of 5 months) and small head circumference for her age. She had a metabolic acidosis and raised plasma lactate and pyruvate concentrations $(3.3 \mathrm{mmol} / 1$, and $0.12 \mathrm{mmol} / 1$, respectively); analysis of her urine showed excessive excretion of 3-hydroxyisovalerate, 3-hydroxybutyrate, succinate, and 3-hydroxy-3-methylglutarate. Plasma biotinidase activity was undetectable.

She was given biotin $5 \mathrm{mg}$ twice a day, and improved within three weeks, further improvement was steady with no recurrence of the respiratory or cutaneous features. Head growth has since been satisfactory but there is some cognitive delay (developmental quotient 38 months at 46 months). There was no evidence of visual problems or of auditory handicap (clinically, with brain stem evoked responses, or on audiometry) either before or after treatment.

\section{CASE 7}

This boy was born in 1982 weighing $3160 \mathrm{~g}$, the first child of unrelated British parents. He was initially well with normal development and could sit unaided at the age of 7 months, but at the age of 8 months he presented with ataxia, hypotonia, seizures, and recurrent respiratory infections. Investigations showed a persistent metabolic acidosis with raised plasma lactate concentration (maximum $4.75 \mathrm{mmol} / \mathrm{l}$ ) and urinary organic acid analysis showed increased 3-hydroxyisovalerate and 3-methylcrotonylglycine. Plasma biotinidase activity was unrecordable. Biotin $10 \mathrm{mg}$ a day resulted in considerable improvement within 24 hours and this continued until all his symptoms resolved apart from a mild limb and truncal ataxia. Alopecia developed immediately after the start of treatment but subsequently resolved. Neurosensory deafness was noted later (this had not been tested before treatment).

Development, apart from some speech delay (probably secondary to deafness), has been within normal limits. Mild limb and truncal ataxia continued to be present at $4 \frac{1}{2}$ years of age and intermittently became more severe, usually in association with minor infections. These exacerbations are resolved by temporarily increasing the biotin dose to $15 \mathrm{mg}$ daily, but compliance may not be good.

\section{CASE 8}

This patient has previously been described by Wallace. ${ }^{20}$ He was born in 1984 weighing $3500 \mathrm{~g}$, and is the full brother of case 7 in this report, the second child of the parents. Biotinidase deficiency was diagnosed presymptomatically in a sample of cord blood and confirmed in a sample of venous blood at 4 weeks of age. Biotin $10 \mathrm{mg}$ daily was started at the age of $101 / 2$ weeks. Hearing and vision seemed to be unaffected before treatment. He developed normally with no symptoms of typical biotinidase deficiency, but when he was $2^{1 / 2}$ years old he was noted to have a mild ataxia though continuing on treatment; compliance, however, may not have been good. At $31 / 2$ years of age his hearing, vision, and coordination are normal.

\section{CASE 9}

This boy was born in 1983 weighing $4120 \mathrm{~g}$, the first child of unrelated English parents. He developed normally during the first year although he was always thought to be excessively clumsy. From about 18 months old he became increasingly unsteady and developed frequent upper respiratory tract and chest infections. At the age of 22 months he was hyperventilating, hypotonic, and grossly ataxic in gait and speech; vision and hearing seemed normal. His hair and skin were normal but there was mild erythema of the conjunctivae. He had a compensated metabolic acidosis with a normal lactate concentration $(1.9 \mathrm{mmol} / \mathrm{l}-$ reference range 0.6 to 2.4$)$ and raised pyruvate concentration $(0 \cdot 18$ $\mathrm{mmol} / \mathrm{l}-$ reference range 0.04 to 0.08 ). Urinary organic acid excretion was abnormal with excessive amounts of 3-methylcrotonate, 3-hydroxyisovalerate and 3-methylcrotonylglycine. Leucocyte carboxylases were decreased (PCC 17\%, MCC 17\%, PC $<1 \%$, all activities expressed as percentages of the means of the normal ranges).

Biotin $10 \mathrm{mg}$ a day was started and his neurological state improved within four days; this continued for several weeks, eventually all the signs completely resolved. After about two months the dose of biotin was reduced to $1 \mathrm{mg}$ a day. $\mathrm{He}$ remained on this regimen for three months when, during a febrile illness, he became mildly ataxic again and the dose of biotin was returned to $10 \mathrm{mg}$ a day. Since then he has remained well and has developed normally but he tends to become ataxic during intercurrent illnesses and his fine movements are rather clumsy. At the age of $3 \frac{1 / 2}{2}$ years his 
hearing was normal, but at 4 years 3 months he was found to be deaf with a neurosensory hearing loss.

CASE 10

This girl was born in 1983, the third child of related Iranian parents. Their first child had died at the age of $1 \frac{1 / 2}{2}$ years of pneumonia and skin lesions, and the second child had died at the age of 1 year of gastroenteritis. Their fourth child seems to be normal.

The patient was well until the age of $1 \frac{1 / 2}{2}$ years when she developed an extensive erythematous rash and alopecia. A tentative diagnosis of acrodermatitis enteropathica was made and zinc supplements were given. Shortly afterwards she developed gastroenteritis, a metabolic acidosis, and exacerbation of her rash. This improved and she was discharged from hospital taking zinc supplements. The rash resolved but recurred at 2 years of age when she again developed a severe metabolic acidosis, fever, signs of cerebellar ataxia, myoclonic jerks, and exaggerated tendon reflexes. Urinary amino acid analysis showed raised concentrations of branched chain amino acids and a diagnosis of intermittent maple syrup urine disease was made. She was discharged on a low protein diet with supplements of Milupa MSUD2; the neurological signs resolved within a month and the rash and alopecia within two months. For the next 18 months she continued on a low protein diet. The rash recurred repeatedly, improving when she was given two scoops of Milupa MSUD2 formula only to reappear after about 12 days. Her development was otherwise normal.

At the age of $31 / 2$ years she was referred from Iran to the United Kingdom where she was found to have mild dermatitis around the nares and was generally hypotonic with brisk tendon reflexes. She had optic atrophy but good visual acuity, and hearing was normal. A clinical diagnosis of biotinidase deficiency was made and confirmed by enzyme assay. Biotin 5 $\mathrm{mg}$ twice a week resulted in complete resolution of the rash within four days and return of muscle tone to normal. (Milupa MSUD2 contains $0.3 \mathrm{mg}$ of free biotin/100 $\mathrm{g}$ and two scoops of formula provides about $30 \mu \mathrm{g}$ of free biotin.)

\section{Discussion}

From the cases described here and from other reports it seems that the presentation of biotinidase deficiency may vary. The onset may be acute or insidious, with either a steady progression or a series of acute incidents interspersed with periods of apparent normality. Hypotonia, ataxia, fits, dermatitis, alopecia, and recurrent respiratory infections are the most common clinical features, but none of these is seen in all cases. Even the metabolic acidosis may not be a constant feature, and urinary organic acid excretion may be unremarkable particularly early in the disease.

Although the immediate response to biotin may be considerable, with complete resolution of the metabolic, skin and, hair abnormalities, it is becoming increasingly obvious that there may be long term complications. Neurosensory deafness and impairment of the optic nerve and visual pathways are now well recognised, and have been described in this report (cases 1,2,3, and 7, and cases 1,2,3, and 10 . respectively). The courses of the patients described here also suggest that intellectual impairment (cases 3 and 6) and persistent ataxia (cases 7, 8 , and 9) may be encountered. The cause of these deficits is not clear, nor is it apparent whether the underlying damage occurs before the start of treatment, though it may not be recognised until later. It is important, however, to note that in one case (case 8) ataxia developed despite presymptomatic treatment with biotin, and that in case 9 neurosensory deafness, which had been shown to be absent initially, developed while the child was receiving regular treatment.

Though this does not preclude the possibility that the underlying damage occurs earlier, it may indicate that the damage continues after treatment has been started and it seems that a more guarded prognosis is necessary even when the child seems normal at the time of diagnosis. Neurological damage may be the result of high concentrations of biocytin in conjunction with low concentrations of biotin, and the biotin dose should therefore be kept to a minimum to lower the rate of turnover of carboxylases and thus the rate of production of biocytin or biotinyl peptides or both. It is of interest that in a few cases low doses of biotin seemed enough to control the metabolic abnormalities, though the other manifestations may not resolve entirely unless higher doses are given (case 10). Other patients require much larger doses and requirements are increased by intercurrent mild illnesses (cases 7 and 9).

In view of the variation in the clinical presentation and of the occurrence of long term complications and the apparent differences in biotin requirements, the possibility that biotinidase may have an additional role (possibly as a biotin transport protein) should be considered. It could then be postulated that different symptoms could be assigned to the deficiency of different functions and that in some cases only one function is lost. The fact that the occurrence of persistent deficits varies within a kinship, however (cases 4 and 6 had no long term visual problems though the related child, case 2 
described by Taitz et al, ${ }^{16}$ developed optic atrophy), seems to disprove this hypothesis.

We thank Dr D Gardner-Medwin (Newcastle), Dr J Insley (Birmingham), Dr M King (Glasgow), Dr JV Leonard (London), Dr VJ Marrian (Perth), Dr R Moore (Belfast), Dr LS Taitz (Sheffield), and Dr SJ Wallace (Cardiff) for allowing us to study their patients.

\section{References}

${ }^{1}$ Moss J, Lane MD. The biotin dependent enzymes. Adv Enzymol 1971;35:321-442.

2 Murthy PNA, Mistry SP. Synthesis of biotin-dependent carboxylases from their apoproteins and biotin. Journal of Scientific and Industrial Research 1972;31:554-63.

${ }^{3}$ Wolf B, Gravel R, Harris DJ, et al. Clinical features of propionic acidemia: variability of expression in 63 patients. Pediatr Res 1979;13:428.

${ }^{4}$ Gravel RA, Robinson BH. Biotin-dependent carboxylase deficiencies (propionyl $\mathrm{CoA}$ and pyruvate carboxylases). Ann NY Acad Sci 1985;447:225-34.

5 Leonard JV, Seakins JWT, Bartlett K, Hyde J, Wilson J, Clayton B. Inherited disorders of 3-methylcrotonyl CoA carboxylation. Arch Dis Child 1981;56:53-9.

${ }^{6}$ Blom W, Muinck Keizer SMPF, Scholte HR. Acetyl CoA carboxylase deficiency: an inborn error of de novo fatty acid synthesis. $N$ Engl J Med 1981;305:465-6.

7 Hommes FA, Polman HA, Reerink JD. Leigh's encephalomyelopathy; an inborn error of gluconeogenesis. Arch Dis Child 1968;43:423-6.

8 Tang TT, Good TA, Dyken PR, et al. Pathogenesis of Leigh's encephalomyelopathy. J Pediatr 1972;81:189-90.

9 Atkin BM, Utter MF, Wienberg MB. Pyruvate carboxylase and phosphoenolpyruvate carboxykinase activity in leukocytes and fibroblasts from a patient with pyruvate carboxylase deficiency. Pediatr Res 1979;13:38-43.

${ }^{10}$ Wolf B, Grier RE, Allen RJ, Goodman SI, Kien CL. Biotinidase deficiency: the enzymatic defect in late-onset multiple carboxylase deficiency. Clin Chim Acta 1983;131:273-81.

11 Burri BJ, Sweetman L, Nyhan WL. Mutant holocarboxylase synthetase. Evidence for the enzyme defect in early infantile biotin responsive multiple carboxylase deficiency. $J$ Clin Invest 1981;68:1491-5.

12 Sweetman L, Burri BJ, Nyhan WL. Biotin holocarboxylase synthetase deficiency. Ann NY Acad Sci 1985;447:288-95.

13 Wolf B, Grier RE, Allen RF, et al. Phenotypic variation in biotinidase deficiency. J Pediatr 1983;103:233-7.

${ }^{14}$ Leonard JV, Bartlett K. Phenotypic variations in Biotinidase deficiency. J Pediatr 1984;104:965.

15 Wolf B, Grier RE, Heard GS. Hearing loss in biotinidase deficiency. Lancet 1983;ii:1365-6.

16 Taitz LS, Leonard JV, Bartlett K. Long term auditory and visual complications of biotinidase deficiency. Early Hum Dev 1985; 11:325-31.

17 Bartlett N, Ng H, Dale G, Green A, Leonard JV. Studies on cultured fibroblasts from patients with defects of biotin dependent carboxylation. J Inherited Metab Dis 1981;4:183-9.

18 Charles BM, Hosking A, Green A, Pollitt R, Bartlett K, Taitz LS. Biotin-responsive alopecia and developmental regression. Lancet 1979;ii:118-20.

19 Taitz LS, Green A, Strachen I, Bartlett K, Bennet M. Biotinidase deficiency and the eye and ear. Lancet 1983;ii:918.

${ }^{20}$ Wallace S. Biotinidase deficiency: presymptomatic treatment. Arch Dis Child 1985;60:574-5.

Correspondence to $\mathrm{Dr} \mathrm{HJ}$ Wastell, Department of Clinical Biochemistry, Newcastle General Hospital, Newcastle upon Tyne NE4 6BE.

Accepted 14 March 1988 